\title{
Alternating current regimes in linear electric circuits according to the relativistic circuit theory
}

\author{
Emil Panov ${ }^{1}$ \\ 1 - Technical University of Varna, Department of Theoretical Electrical Engineering and Instrumentation, 9010, 1 Studentska Street, Varna, \\ Bulgaria \\ Corresponding author contact: eipanov@yahoo.com
}

\begin{abstract}
The paper presents some new chapters of the relativistic circuit theory, which are part of the special theory of relativity. It explores the alternating current regimes in the linear electric circuits, which are moving with very high speeds less than the speed of light. In the paper a large group of basic problems, connected with the relativistic fundamental laws in the time domain and in phasor form for the linear electric circuits are observed. The relativistic forms of the phasors of the basic quantities of the electric circuits (currents, voltages), the complex powers and the relativistic relations of the basic parameters of the circuits (angular frequencies, phases, phase shifts, reactances, susceptances, impedances, admittances) are presented, too. Additionally, some phenomena as resonances and transient processes in fast moving linear electric circuits are observed, as well. All the formulas in the paper are extracted consecutively and they are followed by explanations in full details. The final results are supported by many simple examples about fast moving linear electric circuits.
\end{abstract}

Keywords: relativistic circuit theory, special circuit theory, alternating current regimes in relativistic form, resonance in relativistic form, transient processes in relativistic form

\section{Introduction}

The creation of the electromagnetic (EM) field theory had a great influence on the modern physics (Maxwell, 1861, 1865, 1873). It was the main reason for the appearance of the Special Theory of Relativity (STR) (Einstein, 1905, 1908), (Feynmann, 1964a), (Kittel, 1963), which changed the modern views on the surrounding phenomena. As a consequence of it was the relativistic correction of the Maxwell-Hertz set of equations of the EM field for moving electromagnetic objects (Maxwell, 1873), (Hertz, 1890), (Feynmann, 1964b), (Purcell, 1965), (Simoniy, 1964), (Smythe, 1989) and the result was the creation of the Maxwell-Hertz-Einstein set of equations of the EM field. The appearance of the Rotary Theory (RT) of the EM field established some corrections in that set by the method of moments (Panov, 2015, 2017a, 2017b). For a long time the Circuit Theory (CT), being a complementary theory of the EM theory, was not presented very well in relativistic form. There are some scientists, who believe that: ...'The important consequences... (of STR) ... are related to the sphere of physics, but not to electrical engineering...”... (Simonyi, 1964, p. 725). At the same time there is a group of scientists who present in their monographs some elements of the Relativistic Circuit Theory (RCT) (or the Special Circuit Theory (SCT)) (Pauli, 1958), (Meerovich, 1966), (Polivanov, 1982), (Meerovich, 1987). In (Panov, 2018) a substantial step forward was done trying to present the basic laws, quantities and parameters of the electric circuits in relativistic form.

In the present paper some additional questions connected with the fast moving electric circuits are observed and they are connected with the alternating current (AC) regimes in the electric circuits. In this case a series of basic questions, connected with the relativistic forms of the fundamental laws in the time domain and in phasor form for the linear electric circuits, are discussed. The relativistic forms of the phasors of the currents and the voltages in the electric circuits, the complex powers and the basic parameters of the circuits (angular frequencies, phases, phase shifts, reactances, susceptances, impedances, admittances) are presented, too. Additionally, resonances and transient processes in fast moving linear electric circuits are observed, as well. The main results are illustrated by the help of some simple examples with fast moving electric circuits. 


\section{$2 \quad$ Analysis}

\subsection{Relativistic laws of moving AC electric circuits}

The basic principle of STR, the relativity principle, states that the physical laws preserve their basic forms in each inertial coordinate system (Einstein, 1905). If there is a static Cartesian coordinate system $S$ and another system $S^{\prime}$, which is moving with a speed $\vec{v}=\vec{v}_{x}$ towards the first one along its $x$ axis and if there is a static electric circuit in the last coordinate system $S^{\prime}$, then we can explore the behavior of that circuit, which is moving towards the first coordinate system $S$ (Fig. 1 - Fig. 23). In (Panov, 2018) the relativistic forms of the Kirchhoff's laws for direct current (DC) regimes were presented. The case when AC regimes are observed their forms are similar, but they are related to the instantaneous values of the currents and the voltage drops.

If we introduce the coefficient of relativity as:

$$
\gamma=\frac{1}{\sqrt{1-\frac{v^{2}}{c^{2}}}}
$$

where $c$ is the speed of light in vacuum and $0 \leq v \leq c$ and if we use the transverse and the longitudinal components of the EM quantities according to the notations of Einstein-Laub (Einstein, 1908), both laws receive very simple forms.

Having in mind the connection between the moments of time $t^{\prime}$ and $t$ in both coordinate systems $S^{\prime}$ and $S$ :

$$
t^{\prime}=\frac{t}{\gamma}=t \cdot \sqrt{1-\frac{v^{2}}{c^{2}}}
$$

we can formulate the Kirchhoff's current law for the instantaneous values of the conduction currents in the time domain in the fast moving electric circuits in relativistic form as follows:

$$
\begin{aligned}
& i_{I_{\text {cond. }}}\left(t^{\prime}\right)=\gamma \cdot i_{I_{\text {cond. }}}\left(\frac{t}{\gamma}\right)=\frac{i_{I I}\left(\frac{t}{\gamma}\right)}{\gamma} \\
& i_{\perp_{\text {cond. }}}\left(t^{\prime}\right)=\gamma \cdot i_{\perp_{\text {cond. }}}\left(\frac{t}{\gamma}\right)=\gamma \cdot i_{\perp}\left(\frac{t}{\gamma}\right)
\end{aligned}
$$

In the moving coordinate system $S^{\prime}$ it will look like:

$$
\sum_{k=1}^{n} i_{k}^{\prime}\left(t^{\prime}\right)=0
$$

or

$$
\sum_{k=1}^{n} i_{\text {cond } \cdot k}^{\prime}\left(t^{\prime}\right)=0
$$

or

$$
\sum_{k=1}^{n} i_{\text {II } \text { cond }_{\cdot k}}\left(t^{\prime}\right)+\sum_{k=1}^{n} i_{\perp_{\text {cond } \cdot k}^{\prime}}\left(t^{\prime}\right)=0
$$

In the static coordinate system $S$ it will look like:

$$
\sum_{k=1}^{n} \gamma \cdot i_{k}\left(\frac{t}{\gamma}\right)=0
$$

or

$$
\sum_{k=1}^{n} \gamma \cdot i_{\text {cond } \cdot k}\left(\frac{t}{\gamma}\right)=0
$$


or

$$
\sum_{k=1}^{n} \gamma \cdot i_{I I} \text { cond } \cdot k_{k}\left(\frac{t}{\gamma}\right)+\sum_{k=1}^{n} \gamma \cdot i_{\perp} \text { cond } \cdot k\left(\frac{t}{\gamma}\right)=0
$$

The relativistic relations among the last eight equations are as follows:

$$
\sum_{k=1}^{n} \gamma \cdot i_{I I \text { cond }_{k} k}\left(\frac{t}{\gamma}\right)+\sum_{k=1}^{n} \gamma \cdot i_{\perp_{\text {cond } \cdot k}}\left(\frac{t}{\gamma}\right)=\sum_{k=1}^{n} \frac{i_{\text {II }}\left(\frac{t}{\gamma}\right)}{\gamma}+\sum_{k=1}^{n} \gamma \cdot i_{\perp_{k}}\left(\frac{t}{\gamma}\right)=\sum_{k=1}^{n} i_{\text {IIcond } \cdot k}^{\prime}\left(t^{\prime}\right)+\sum_{k=1}^{n} i_{\perp \text { cond }{ }_{k}}^{\prime}\left(t^{\prime}\right)=0
$$

Here, $i_{\text {cond.k }}^{\prime}$ or $i_{\text {cond.k }}$ is an instantaneous conduction current in the coordinate systems $S^{\prime}$ or $S$, correspondingly and:

$$
i_{I I I_{k}}(t)=i_{\text {II } \text { cond }_{\cdot k}}(t)+i_{\text {II } \text { conv } \cdot k_{k}}(t)
$$

where $i_{I I \text { conv }_{\cdot k}}(t)$ is an instantaneous convection current in the coordinate system $S$. So, equation (11) depicts the relativistic Kirchhoff's current law. The main meaning is connected with the fact that the sum of the instantaneous conduction currents equals zero in each inertial coordinate system.

If we use similar reasoning, it is possible to write down the relativistic connections among the voltage drops in the coordinate systems $S^{\prime}$ and $S$ :

$$
\begin{aligned}
& u_{I I_{\text {cond. }}^{\prime}}\left(t^{\prime}\right)=\gamma \cdot u_{I I_{\text {cond }} .}\left(\frac{t}{\gamma}\right)=\gamma \cdot u_{I I}\left(\frac{t}{\gamma}\right) \\
& u_{\perp_{\text {cond. }}}\left(t^{\prime}\right)=\gamma \cdot u_{\perp_{\text {cond }} .}\left(\frac{t}{\gamma}\right)=\frac{u_{\perp}\left(\frac{t}{\gamma}\right)}{\gamma}
\end{aligned}
$$

Using the last two equations we can formulate the Kirchhoff's voltage law in relativistic form in the time domain. In the moving coordinate system $S^{\prime}$ it will look like:

$$
\sum_{k=1}^{n} u_{k}^{\prime}\left(t^{\prime}\right)=0
$$

or

$$
\sum_{k=1}^{n} u_{\text {cond } \cdot k}^{\prime}\left(t^{\prime}\right)=0
$$

or

$$
\sum_{k=1}^{n} u_{I I}^{\prime} \text { cond } \cdot k_{k}\left(t^{\prime}\right)+\sum_{k=1}^{n} u_{\perp \text { cond } \cdot k}^{\prime}\left(t^{\prime}\right)=0
$$

In the static coordinate system $S$ it will look like:

$$
\sum_{k=1}^{n} \gamma \cdot u_{k}\left(\frac{t}{\gamma}\right)=0
$$

or

$$
\sum_{k=1}^{n} \gamma \cdot u_{\text {cond } \cdot k}\left(\frac{t}{\gamma}\right)=0
$$

or

$$
\sum_{k=1}^{n} \gamma \cdot u_{\text {II } \text { cond } \cdot k}\left(\frac{t}{\gamma}\right)+\sum_{k=1}^{n} \gamma \cdot u_{\perp \text { cond }_{\cdot k}}\left(\frac{t}{\gamma}\right)=0
$$

The relativistic relations among the last eight equations are as follows:

$$
\sum_{k=1}^{n} \gamma \cdot u_{I I \text { cond }_{k}}\left(\frac{t}{\gamma}\right)+\sum_{k=1}^{n} \gamma \cdot u_{\perp \text { cond }_{k}}\left(\frac{t}{\gamma}\right)=\sum_{k=1}^{n} \gamma \cdot u_{I I_{k}}\left(\frac{t}{\gamma}\right)+\sum_{k=1}^{n} \frac{u_{\perp_{k}}\left(\frac{t}{\gamma}\right)}{\gamma}=\sum_{k=1}^{n} u_{\text {IIcond }}^{\prime}\left(t^{\prime}\right)+\sum_{k=1}^{n} u_{\perp \text { cond }_{k}}^{\prime}\left(t^{\prime}\right)=0
$$


In the last few equations $u_{c o n d_{. k}}^{\prime}$ or $u_{\text {cond.k }}$ is a voltage, which is the reason for the flowing of a conduction current. Except that:

$$
u_{\perp_{k}}(t)=u_{\perp_{\text {cond } \cdot k}}(t)+u_{\perp_{\text {ind } \cdot k}}(t)
$$

At the same time the voltage $u_{\perp_{i n d_{-k}}}(t)$ is a result of the unipolar induction. Here, equation (21) depicts the relativistic Kirchhoff's voltage law. The main meaning is connected with the fact that the sum of the instantaneous voltage drops in a contour of a circuit equals zero in each inertial coordinate system.

The integral form of Joule's law for a finite longitudinal or transverse element of a conductor with conduction current is as follows:

$$
\begin{aligned}
& P_{I I}^{\prime}\left(t^{\prime}\right)=u_{I I_{\text {cond. }}^{\prime}}\left(t^{\prime}\right) \cdot i_{I_{\text {cond. }}^{\prime}}\left(t^{\prime}\right)=\left[\gamma \cdot u_{I I_{\text {cond. }}}\left(\frac{t}{\gamma}\right)\right] \cdot\left[\gamma \cdot i_{I_{\text {cond. }}}\left(\frac{t}{\gamma}\right)\right]=\gamma^{2} \cdot u_{I I_{\text {cond. }}}\left(\frac{t}{\gamma}\right) \cdot i_{I I_{\text {cond. }}}\left(\frac{t}{\gamma}\right)=\gamma^{2} \cdot P_{I I}\left(\frac{t}{\gamma}\right) \\
& P_{\perp}^{\prime}\left(t^{\prime}\right)=u_{\perp_{\text {cond. }}^{\prime}}\left(t^{\prime}\right) \cdot i_{\perp_{\text {cond. }}^{\prime}}\left(t^{\prime}\right)=\left[\gamma \cdot u_{\perp_{\text {cond. }}}\left(\frac{t}{\gamma}\right)\right] \cdot\left[\gamma \cdot i_{\perp_{\text {cond. }}}\left(\frac{t}{\gamma}\right)\right]=\gamma^{2} \cdot u_{\perp_{\text {cond. }}}\left(\frac{t}{\gamma}\right) \cdot i_{\perp_{\text {cond. }}}\left(\frac{t}{\gamma}\right)=\gamma^{2} \cdot P_{\perp}\left(\frac{t}{\gamma}\right)
\end{aligned}
$$

where $P_{I I}^{\prime}, P_{I I}, P_{\perp}^{\prime}$ and $P_{\perp}$ are the instantaneous powers, caused by the conduction currents in the coordinate systems $S^{\prime}$ and $S$. Relations among the powers in the coordinate systems $S^{\prime}$ and $S$ are also presented in (Pauli, 1958).

The integral form of Ohm's law for a finite longitudinal or transverse element of a conductor with conduction current is as follows:



$$
\begin{aligned}
& u_{\perp_{\text {cond. }}^{\prime}}\left(t^{\prime}\right)=R_{\perp}^{\prime} \cdot i_{\perp_{\text {cond. }}^{\prime}}\left(t^{\prime}\right)=R_{\perp} \cdot\left[\gamma \cdot i_{\perp_{\text {cond. }}}\left(\frac{t}{\gamma}\right)\right]=\gamma \cdot R_{\perp} \cdot i_{\perp_{\text {cond }} .}\left(\frac{t}{\gamma}\right)=\gamma \cdot u_{\perp_{\text {cond. }}}\left(\frac{t}{\gamma}\right)=\frac{u_{\perp}\left(\frac{t}{\gamma}\right)}{\gamma}
\end{aligned}
$$

where $R_{I I}^{\prime}, R_{I I}, R_{\perp}^{\prime}$ and $R_{\perp}$ are the resistances of the longitudinal or the transverse element of a conductor in the coordinate systems $S^{\prime}$ and $S$. Some relations among the parameters of the resistances in both coordinate systems are presented in (Meerovich, 1966).

\subsection{Basic relations of the parameters of fast moving AC linear electric circuits}

In (Panov, 2018) the relations among the resistances and the coductances of the conductors of fast moving circuits in the coordinate systems $S^{\prime}$ and $S$ were extracted:

$$
\begin{aligned}
& R_{I I}^{\prime}=\frac{1}{G_{I I}^{\prime}}=R_{I I}=\frac{1}{G_{I I}} \\
& R_{\perp}^{\prime}=\frac{1}{G_{\perp}^{\prime}}=R_{\perp}=\frac{1}{G_{\perp}}
\end{aligned}
$$

Here, $G_{I I}^{\prime}, G_{I I}, G_{\perp}^{\prime}$ and $G_{\perp}$ are the conductances of a longitudinal or a transverse element of a conductor in the coordinate systems $S^{\prime}$ and $S$.

In (Panov, 2018) another important relations were extracted for the capacitances $\left(C_{I I}^{\prime}, C_{I I}, C_{\perp}^{\prime}\right.$, $\left.C_{\perp}\right)$ and the inductances $\left(L_{I I}^{\prime}, L_{I I}, L_{\perp}^{\prime}, L_{\perp}\right)$ of the reactive elements of the explored electric circuits in the coordinate systems $S^{\prime}$ and $S$ :

$$
C_{I I}^{\prime}=\frac{C_{I I}}{\gamma}
$$




$$
\begin{aligned}
& C_{\perp}^{\prime}=\frac{C_{\perp}}{\gamma} \\
& L_{I I}^{\prime}=\frac{L_{I I}}{\gamma} \\
& L_{\perp}^{\prime}=\frac{L_{\perp}}{\gamma}
\end{aligned}
$$

The instantaneous values of the stored energy of a charged capacitor $\left(W_{C_{I I}}^{\prime}, W_{C_{I I}}, W_{C_{\perp}}^{\prime}, W_{C_{\perp}}\right)$ and a coil with a current $\left(W_{L_{I I}}^{\prime}, W_{L_{I I}}, W_{L_{\perp}}^{\prime}, W_{L_{\perp}}\right)$ in both coordinate systems in case of AC regimes have the following forms:

$$
\begin{gathered}
W_{C_{I I}}^{\prime}\left(t^{\prime}\right)=\frac{C_{I I}^{\prime} \cdot\left[u_{C_{I I}^{\prime}}\left(t^{\prime}\right)\right]^{2}}{2}=\frac{\gamma \cdot C_{I I} \cdot\left[u_{L_{I I}}\left(\frac{t}{\gamma}\right)\right]^{2}}{2}=\gamma \cdot W_{C_{I I}}\left(\frac{t}{\gamma}\right) \\
W_{C_{\perp}}^{\prime}\left(t^{\prime}\right)=\frac{C_{\perp}^{\prime} \cdot\left[u_{C_{\perp}}^{\prime}\left(t^{\prime}\right)\right]^{2}}{2}=\frac{\gamma \cdot C_{\perp} \cdot\left[u_{L_{\perp}}\left(\frac{t}{\gamma}\right)\right]^{2}}{2}=\gamma \cdot W_{C_{\perp}}\left(\frac{t}{\gamma}\right) \\
W_{L_{I I}}^{\prime}\left(t^{\prime}\right)=\frac{L_{I I}^{\prime} \cdot\left[i^{\prime} L_{I I}\left(t^{\prime}\right)\right]^{2}}{2}=\frac{\gamma \cdot L_{I I} \cdot\left[i_{L_{I I}}\left(\frac{t}{\gamma}\right)\right]^{2}}{2}=\gamma \cdot W_{L_{I I}}\left(\frac{t}{\gamma}\right) \\
W_{L_{\perp}}^{\prime}\left(t^{\prime}\right)=\frac{L_{\perp}^{\prime} \cdot\left[i_{L_{\perp}}\left(t^{\prime}\right)\right]^{2}}{2}=\frac{\gamma \cdot L_{\perp} \cdot\left[i_{L_{\perp}}\left(\frac{t}{\gamma}\right)\right]^{2}}{2}=\gamma \cdot W_{L_{\perp}}\left(\frac{t}{\gamma}\right)
\end{gathered}
$$

The last four equations are received by the help of equations (25), (26), (29), (30), (31) and (32).

In order to introduce the basic relations among the rest of the parameters of the fast moving electric circuits as the angular frequencies, the phases, the phase shifts, the reactances, the susceptances, the impedances and the admittances of the elements of these circuits, it is better to start with some simple examples of such AC electric circuits in the coordinate systems $S^{\prime}$ and $S$.

\subsection{Time domain analysis and phasor approach of fast moving AC linear electric circuits}

In Fig. 1 a serial RL electric circuit is presented, which is operating in harmonic regime and at the same time it is moving with a uniform speed $\vec{v}_{x}$ along the $x$-axis of a static Cartesian coordinate system $S$. It can be explored also in the moving coordinate system $S^{\prime}$, towards which the circuit is in a static position. We need to analyze the basic quantities and parameters of that fast moving electric circuit in both coordinate systems firstly in time domain and then by the help of the phasor approach in the Gaussian complex plane. 



(b)

Fig. 1. A moving serial RL electric circuit with a uniform speed $\vec{v}_{x}$ towards a static Cartesian coordinate system $S$.

If the electromotive force (e.m.f.) of the voltage source has a zero phase shift, then the current in the coordinate system $S$ ' will be:

$$
i^{\prime}\left(t^{\prime}\right)=i_{p}^{\prime} . \sin \left(\omega^{\prime} \cdot t^{\prime}-\alpha^{\prime}\right)=\frac{e_{p}^{\prime}}{\sqrt{R^{\prime 2}+\left(\omega^{\prime} \cdot L^{\prime}\right)^{2}}} \cdot \sin \left(\omega^{\prime} \cdot t^{\prime}-\operatorname{arctg} \frac{\omega^{\prime} \cdot L^{\prime}}{R^{\prime}}\right)
$$

where $i_{p}^{\prime}$ and $e^{\prime}$ are peak values of the current and the e.m.f. In the coordinate system $S$ that current will have the following form:

$$
\begin{aligned}
& i^{\prime}\left(t^{\prime}\right)=i_{p}^{\prime} . \sin \left(\omega^{\prime} \cdot t^{\prime}-\alpha^{\prime}\right)=\frac{e_{p}^{\prime}}{\sqrt{R^{\prime 2}+\left(\omega^{\prime} \cdot L^{\prime}\right)^{2}}} \cdot \sin \left(\omega^{\prime} \cdot t^{\prime}-\operatorname{arctg} \frac{\omega^{\prime} \cdot L^{\prime}}{R^{\prime}}\right)= \\
& =\frac{\gamma \cdot e_{p}}{\sqrt{R^{2}+\left(\gamma \cdot \omega \cdot \frac{L}{\gamma}\right)^{2}}} \cdot \sin \left(\gamma \cdot \omega \cdot \frac{t}{\gamma}-\operatorname{arctg} \frac{\gamma \cdot \omega \cdot \frac{L}{\gamma}}{R}\right)=\frac{\gamma \cdot e_{p}}{\sqrt{R^{2}+(\omega \cdot L)^{2}}} \cdot \sin \left(\omega \cdot t-\operatorname{arctg} \frac{\omega \cdot L}{R}\right)= \\
& =\gamma \cdot i_{p} . \sin (\omega \cdot t-\alpha)=\gamma \cdot i\left(\frac{t}{\gamma}\right)
\end{aligned}
$$

from where it is well seen that the peak values of the voltages and the currents are transformed according to formulas (3), (4), (13) and (14). The relations among the angular frequencies, the phase shifts and the phases are as follows:

$$
\begin{gathered}
\omega^{\prime}=\frac{2 . \pi}{T^{\prime}}=\frac{2 . \pi}{\frac{T}{\gamma}}=\gamma \cdot \frac{2 . \pi}{T}=\gamma \cdot \omega \\
\alpha^{\prime}=\alpha \\
\varphi^{\prime}\left(t^{\prime}\right)=\omega^{\prime} \cdot t^{\prime}-\alpha^{\prime}=\omega \cdot t-\alpha=\varphi(t)
\end{gathered}
$$

Equation (39) corresponds to the result from the transverse Doppler effect (Kittel, 1963, p. 344).

In Fig. 2 a simple serial RC electric circuit is presented, which is operating in harmonic regime and which is moving towards the coordinate system $S$. If the electromotive force (e.m.f.) of the voltage source has a zero phase shift, then the current in the coordinate system $S$ ' will be: 



(b)

Fig. 2. A moving serial RC electric circuit with a uniform speed $\vec{v}_{x}$ towards a static Cartesian coordinate system $S$.

$$
i^{\prime}\left(t^{\prime}\right)=i_{p}^{\prime} . \sin \left(\omega^{\prime} \cdot t^{\prime}+\alpha^{\prime}\right)=\frac{e_{p}^{\prime}}{\sqrt{R^{\prime 2}+\left(\frac{1}{\omega^{\prime} \cdot C^{\prime}}\right)^{2}}} \cdot \sin \left(\omega^{\prime} \cdot t^{\prime}+\operatorname{arctg} \frac{1}{\omega^{\prime} \cdot R^{\prime} \cdot C^{\prime}}\right)
$$

In the coordinate system $S$ that current will have the following form:

$$
\begin{aligned}
& i^{\prime}\left(t^{\prime}\right)=i_{p}^{\prime} . \sin \left(\omega^{\prime} \cdot t^{\prime}+\alpha^{\prime}\right)=\frac{e_{p}^{\prime}}{\sqrt{R^{\prime 2}+\left(\frac{1}{\omega^{\prime} \cdot C^{\prime}}\right)^{2}}} \cdot \sin \left(\omega^{\prime} \cdot t^{\prime}+\operatorname{arctg} \frac{1}{\omega^{\prime} \cdot R^{\prime} \cdot C^{\prime}}\right)=
\end{aligned}
$$



$$
\begin{aligned}
& \sqrt{R^{2}+\left(\frac{1}{\gamma \cdot \omega \cdot \frac{C}{\gamma}}\right)} \\
& =\gamma \cdot i_{p} \cdot \sin (\omega \cdot t+\alpha)=\gamma \cdot i\left(\frac{t}{\gamma}\right)
\end{aligned}
$$

The magnitudes of the impedances of the circuits presented in Fig. 1 and Fig. 2 in both coordinate systems $S^{\prime}$ and $S$ preserve their values:

$$
\begin{gathered}
z^{\prime}=\sqrt{R^{\prime 2}+\left(\omega^{\prime} \cdot L^{\prime}\right)^{2}}=\sqrt{R^{\prime 2}+X_{L^{\prime}}^{\prime 2}}=\sqrt{R^{2}+(\omega \cdot L)^{2}}=\sqrt{R^{2}+X_{L}^{2}}=z \\
z^{\prime}=\sqrt{R^{\prime 2}+\left(\frac{1}{\omega^{\prime} \cdot C^{\prime}}\right)^{2}}=\sqrt{R^{\prime 2}+X_{C^{\prime}}^{\prime 2}}=\sqrt{R^{2}+\left(\frac{1}{\omega \cdot C}\right)^{2}}=\sqrt{R^{2}+X_{C}^{2}}=z
\end{gathered}
$$

The triangles of the magnitudes of the impedances of both circuits (Fig. 3 and Fig. 4) preserve their forms and scales in the coordinate systems $S^{\prime}$ and $S$. Here, it is accepted for simplicity that the value of the phase shift of the current in the coordinate systems $S^{\prime}$ is zero. 

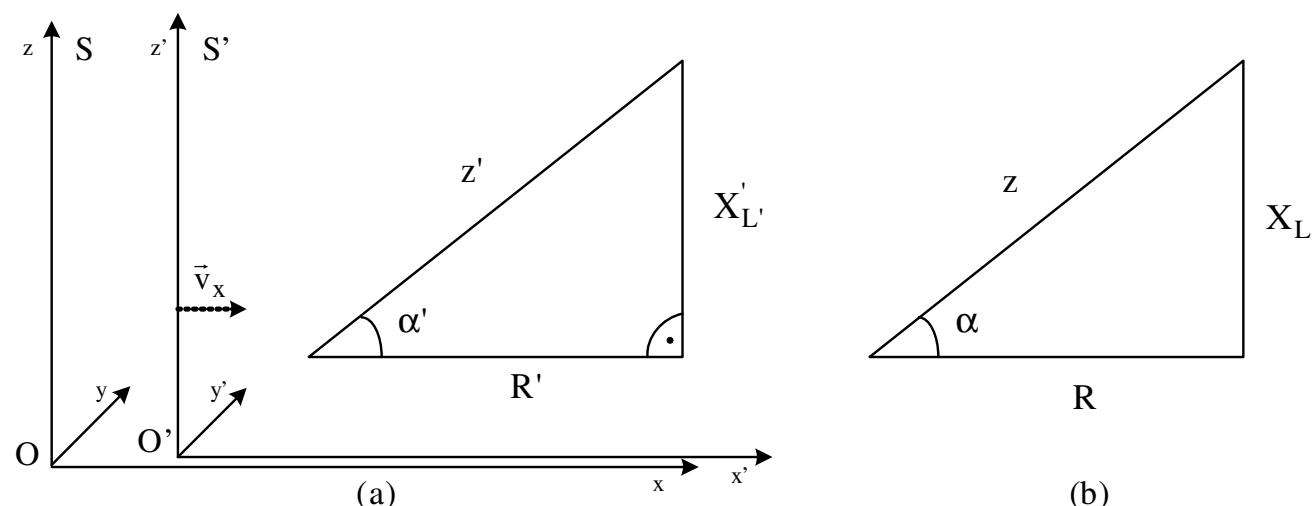

(b)

Fig. 3. Triangles of the impedances of a moving serial RL electric circuit with a uniform speed $\vec{v}_{x}$ towards a static Cartesian coordinate system $S$.

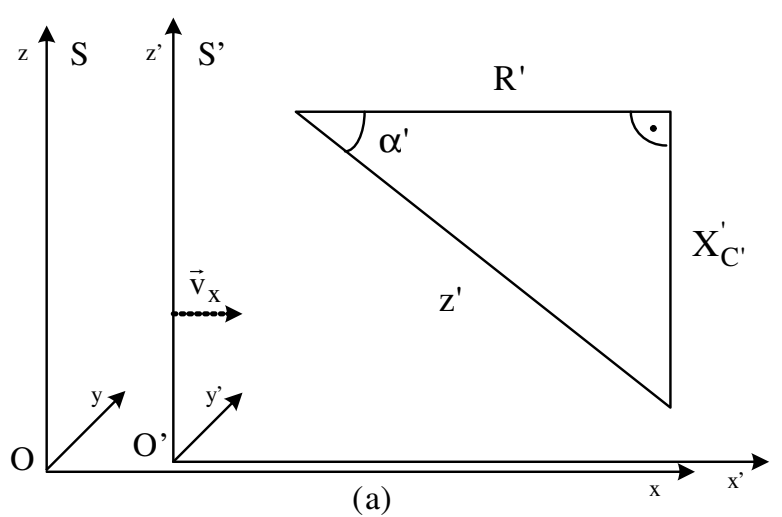

(a)

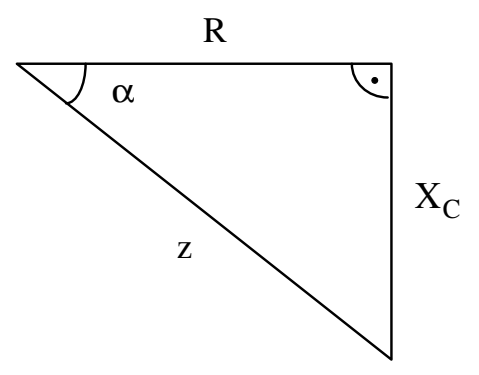

(b)

Fig. 4. Triangles of the impedances of a moving serial RC electric circuit with a uniform speed $\vec{v}_{x}$ towards a static Cartesian coordinate system $S$.

The real powers, the reactive powers and the apparent powers of both circuits in the coordinate systems $S$ ' and $S$ are connected with the following relations:

$$
\begin{aligned}
& S^{\prime}=E^{\prime} \cdot I^{\prime}=\sqrt{P_{R^{\prime}}^{\prime 2}+Q_{L^{\prime}}^{\prime 2}}=\sqrt{\left(U_{R^{\prime}}^{\prime} \cdot I^{\prime}\right)^{2}+\left(U_{L^{\prime}}^{\prime} \cdot I^{\prime}\right)^{2}}=\sqrt{\left(E^{\prime} \cdot I^{\prime} \cdot \cos \alpha^{\prime}\right)^{2}+\left(E^{\prime} \cdot I^{\prime} \cdot \sin \alpha^{\prime}\right)^{2}}= \\
& =\gamma \cdot E \cdot \gamma \cdot I=\gamma^{2} \cdot E \cdot I=\sqrt{(\gamma \cdot E \cdot \gamma \cdot I \cdot \cos \alpha)^{2}+(\gamma \cdot E \cdot \gamma \cdot I \cdot \sin \alpha)^{2}}=\sqrt{\left(\gamma^{2} \cdot E \cdot I \cdot \cos \alpha\right)^{2}+\left(\gamma^{2} \cdot E \cdot I \cdot \sin \alpha\right)^{2}}=(46) \\
& =\sqrt{\left(\gamma^{2} \cdot P_{R}\right)^{2}+\left(\gamma^{2} \cdot Q_{L}\right)^{2}}=\gamma^{2} \cdot \sqrt{P_{R}^{2}+Q_{L}^{2}}=\gamma^{2} \cdot S \\
& S^{\prime}=E^{\prime} \cdot I^{\prime}=\sqrt{P_{R^{\prime}}^{\prime 2}+Q_{C^{\prime}}^{\prime 2}}=\sqrt{\left(U_{R^{\prime}}^{\prime} \cdot I^{\prime}\right)^{2}+\left(U_{C^{\prime}}^{\prime} \cdot I^{\prime}\right)^{2}}=\sqrt{\left(E^{\prime} \cdot I^{\prime} \cdot \cos \alpha^{\prime}\right)^{2}+\left(E^{\prime} \cdot I^{\prime} \cdot \sin \alpha^{\prime}\right)^{2}}= \\
& =\gamma \cdot E \cdot \gamma \cdot I=\gamma^{2} \cdot E \cdot I=\sqrt{(\gamma \cdot E \cdot \gamma \cdot I \cdot \cos \alpha)^{2}+(\gamma \cdot E \cdot \gamma \cdot I \cdot \sin \alpha)^{2}}=\sqrt{\left(\gamma^{2} \cdot E \cdot I \cdot \cos \alpha\right)^{2}+\left(\gamma^{2} \cdot E \cdot I \cdot \sin \alpha\right)^{2}}=(47) \\
& =\sqrt{\left(\gamma^{2} \cdot P_{R}\right)^{2}+\left(\gamma^{2} \cdot Q_{C}\right)^{2}}=\gamma^{2} \cdot \sqrt{P_{R}^{2}+Q_{C}^{2}}=\gamma^{2} \cdot S
\end{aligned}
$$


These two equations express also the balance of powers for these two electric circuits. The final result is connected as well with the power triangles in both coordinate systems $S$ ' and $S$, which preserve their forms, but they don't preserve their scales (Fig. 5 and Fig. 6).

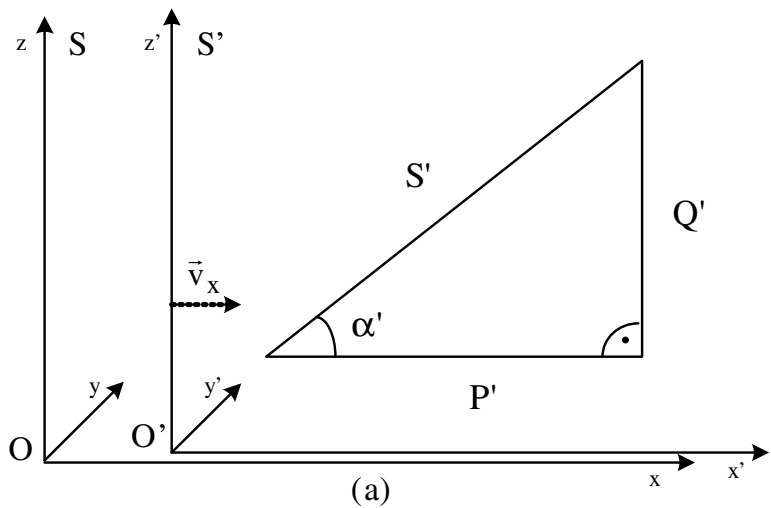

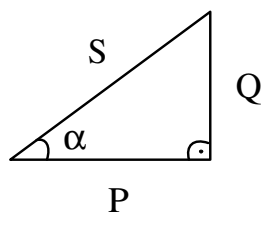

(b)

Fig. 5. Triangles of the powers of a moving serial RL electric circuit with a uniform speed $\vec{v}_{x}$ towards a static Cartesian coordinate system $S$.

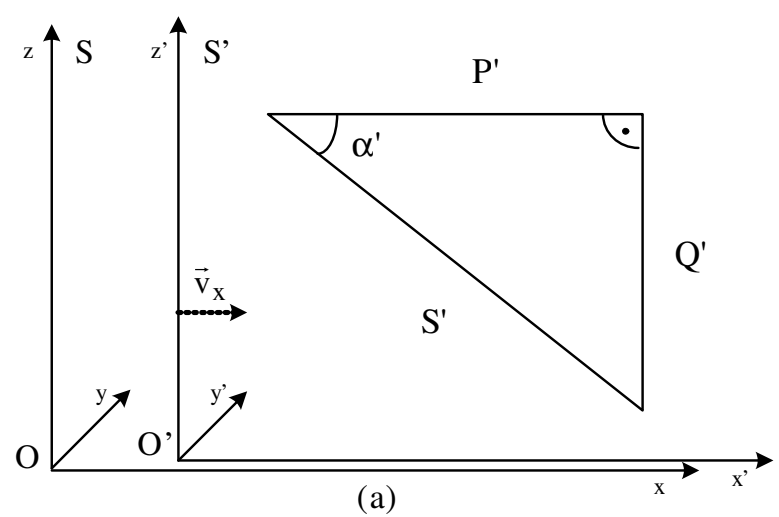

(a)

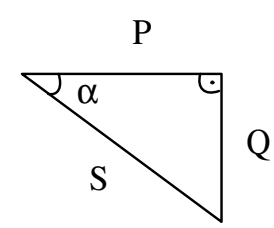

(b)

Fig. 6. Triangles of the powers of a moving serial RC electric circuit with a uniform speed $\vec{v}_{x}$ towards a static Cartesian coordinate system $S$.

It is well known that the phasor approach is a very appropriate transform (operational) method for analysis of linear electric circuit operating in harmonic regimes. Having in mind equations (3), (4), (13), (14), (39), (40) and (41), the basic relations among the quantities (currents, voltages, powers) in phasor form can be extracted and they are as follows:

$$
\begin{gathered}
\dot{I^{\prime}}=I^{\prime} \cdot e^{j \psi^{\prime}}=\gamma \cdot I \cdot e^{j \psi}=\gamma \cdot \dot{I} \\
\dot{U^{\prime}}=U^{\prime} \cdot e^{j \theta^{\prime}}=\gamma \cdot I \cdot e^{j \theta}=\gamma \cdot \dot{U} \\
\dot{S^{\prime}}=\dot{U}^{\prime} \cdot{ }^{*} I^{\prime}=U^{\prime} \cdot e^{j \theta^{\prime}} \cdot I^{\prime} \cdot e^{-j \psi^{\prime}}=U^{\prime} \cdot I^{\prime} \cdot e^{j\left(\theta^{\prime}-\psi^{\prime}\right)}=S^{\prime} \cdot e^{j\left(\theta^{\prime}-\psi^{\prime}\right)}=\gamma \cdot U \cdot e^{j \theta} \cdot \gamma \cdot I \cdot e^{-j \psi}= \\
=\gamma^{2} \cdot U \cdot I \cdot e^{j(\theta-\psi)}=\gamma^{2} \cdot S \cdot e^{j(\theta-\psi)}=\gamma \cdot \dot{U} \cdot{ }^{*} \cdot I^{2}=\gamma^{2} \cdot \dot{U} \cdot I^{*}=\gamma^{2} \cdot \dot{S}
\end{gathered}
$$

Here, $j=\sqrt{-1}$ is the imaginary unit; the currents and the voltages are presented with their root-meansquare (r.m.s.) values; $\dot{S}^{\prime}$ and $\dot{S}$ are complex powers in both coordinate systems $S^{\prime}$ and $S ; I^{\prime}$ and $\stackrel{*}{I}$ are the conjugate values of the phasors $\dot{I}^{\prime}$ and $\dot{I}$. The operator of rotation in positive direction preserves its value: 


$$
e^{j \omega^{\prime} \cdot t^{\prime}}=e^{j \omega \cdot t}
$$

The same fact is valid for the impedances and for the admittances of the elements of the circuits:

$$
\begin{gathered}
Z^{\prime}=z^{\prime} \cdot e^{j \alpha^{\prime}}=z \cdot e^{j \cdot \alpha}=Z \\
Y^{\prime}=\frac{1}{Z^{\prime}}=\frac{1}{z^{\prime} \cdot e^{j \alpha^{\prime}}}=y^{\prime} \cdot e^{-j \alpha^{\prime}}=y^{\prime} \cdot e^{j \delta^{\prime}}=\frac{1}{Z}=\frac{1}{z \cdot e^{j \alpha}}=y \cdot e^{-j \alpha}=y \cdot e^{j \delta}=Y
\end{gathered}
$$

The equivalent circuits of the networks, given in Fig. 1 and Fig. 2, in phasor form are presented in Fig. 7 and Fig. 8, correspondingly.

The vector diagrams of both circuits are presented in Fig. 9 and Fig. 10. These vector diagrams present Kirchhoff's voltage law in phasor form graphically:

$$
\begin{aligned}
& \dot{E^{\prime}}=\dot{U}^{\prime} R^{\prime}+\dot{U}^{\prime} L^{\prime}=\gamma \cdot \dot{U}_{R}+\gamma \cdot \dot{U}_{L}=\gamma \cdot \dot{E} \\
& \dot{E}^{\prime}=\dot{U}^{\prime}{ }_{R^{\prime}}+\dot{U}^{\prime}{ }_{C^{\prime}}=\gamma \cdot \dot{U}_{R}+\gamma \cdot \dot{U}_{C}=\gamma \cdot \dot{E}
\end{aligned}
$$
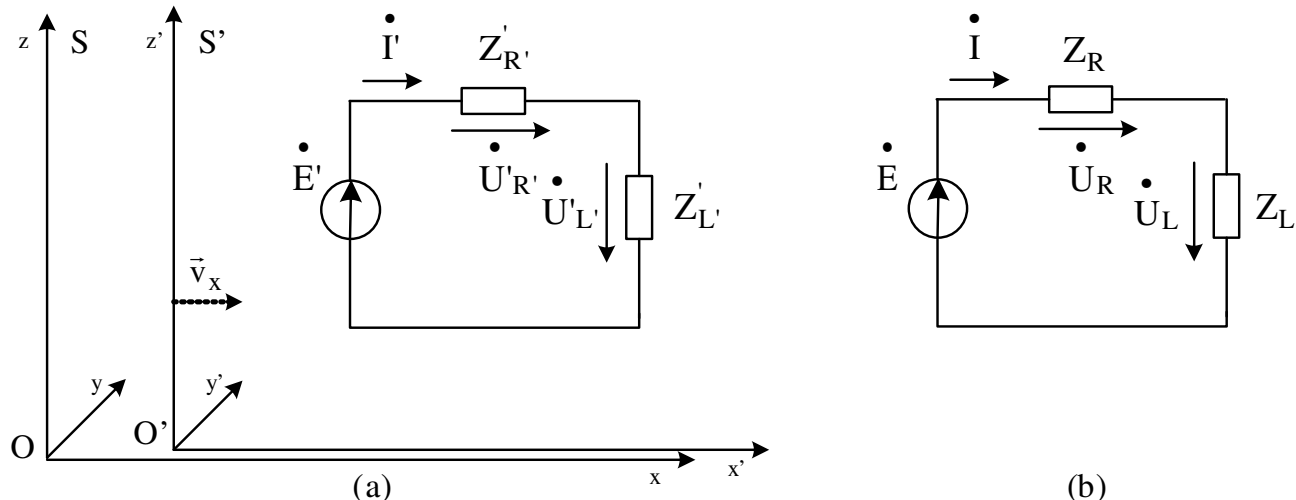

(a)

(b)

Fig. 7. Equivalent circuit of a moving serial RL electric circuit with a uniform speed $\vec{v}_{x}$ towards a static Cartesian coordinate system $S$ according to the phasor approach.

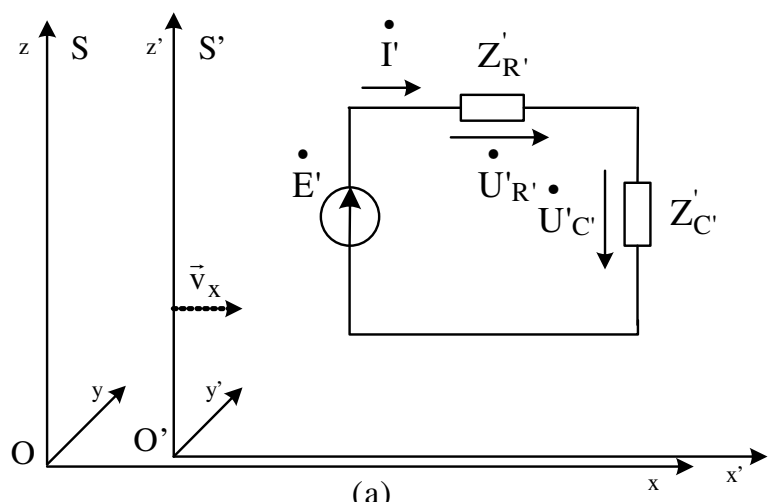

(a)

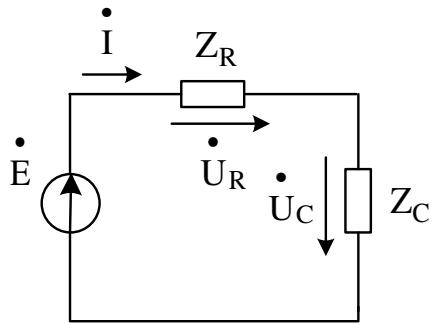

(b)

Fig. 8. Equivalent circuit of a moving serial RC electric circuit with a uniform speed $\vec{v}_{x}$ towards a static Cartesian coordinate system $S$ according to the phasor approach. 


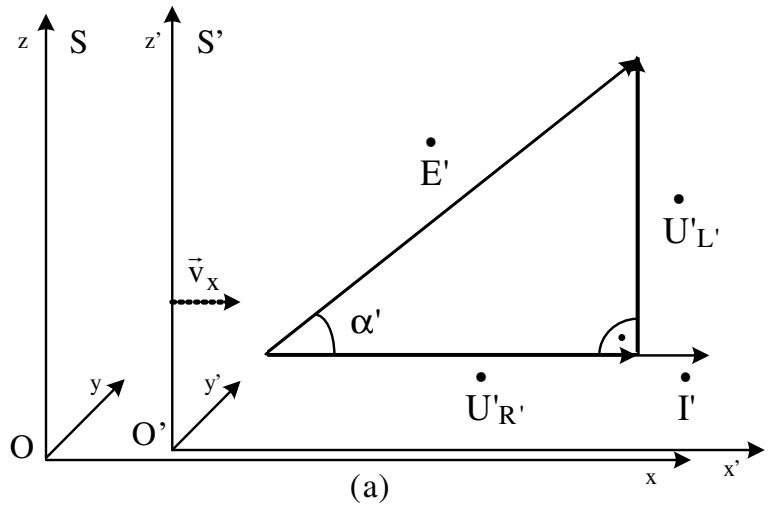

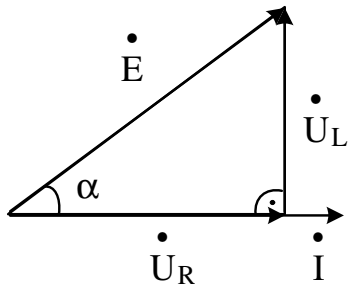

(b)

Fig. 9. Vector diagram of a moving serial RL electric circuit with a uniform speed $\vec{v}_{x}$ towards a static Cartesian coordinate system $S$ according to the phasor approach.

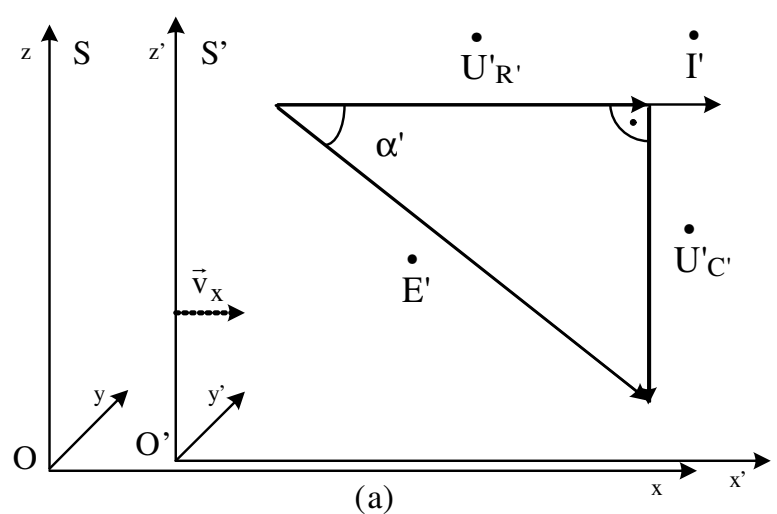

(a)

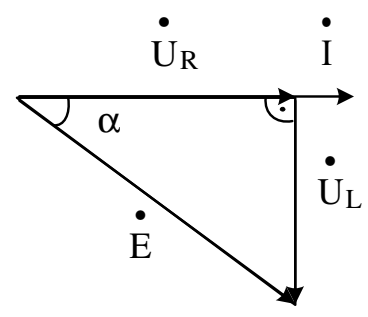

(b)

Fig. 10. Vector diagram of a moving serial RC electric circuit with a uniform speed $\vec{v}_{x}$ towards a static Cartesian coordinate system $S$ according to the phasor approach.

In case of fast moving parallel electric circuits, the results are similar to these, which were extracted so far. In Fig. 11 a parallel RL circuit is presented, and in Fig. 12 a parallel RC circuit is given, too.

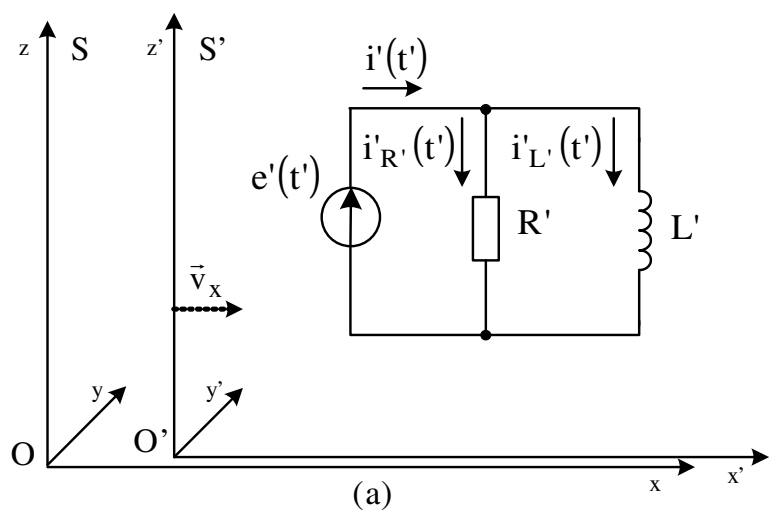

(a)

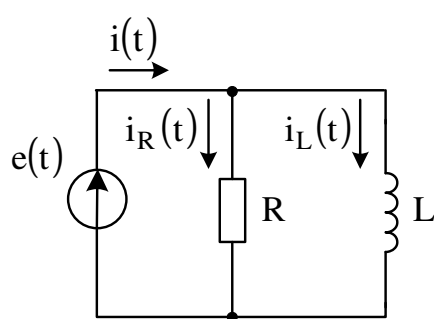

(b)

Fig. 11. A moving parallel RL electric circuit with a uniform speed $\vec{v}_{x}$ towards a static Cartesian coordinate system $S$. 




(a)

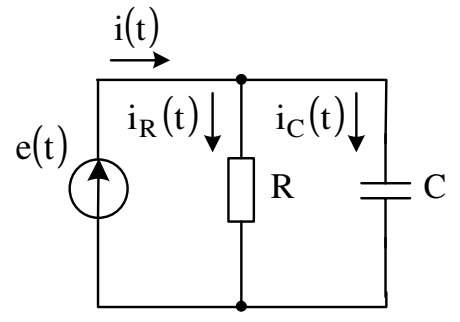

(b)

Fig. 12. A moving parallel RC electric circuit with a uniform speed $\vec{v}_{x}$ towards a static Cartesian coordinate system $S$.

If the electromotive forces (e.m.f.) of the voltage sources have zero phase shifts, then the input currents in the coordinate systems $S$ ' and $S$ will be:

$$
\begin{aligned}
& i^{\prime}\left(t^{\prime}\right)=i_{p}^{\prime} \cdot \sin \left(\omega^{\prime} \cdot t^{\prime}-\alpha^{\prime}\right)=\sqrt{\left(\frac{1}{R^{\prime}}\right)^{2}+\left(\frac{1}{\omega^{\prime} \cdot L^{\prime}}\right)^{2}} \cdot e_{p}^{\prime} \cdot \sin \left(\omega^{\prime} \cdot t^{\prime}-\operatorname{arctg} \frac{R^{\prime}}{\omega^{\prime} \cdot L^{\prime}}\right)= \\
& =\gamma \cdot \sqrt{\left(\frac{1}{R}\right)^{2}+\left(\frac{1}{\omega \cdot L}\right)^{2}} \cdot e_{p} \cdot \sin \left(\omega \cdot t-\operatorname{arctg} \frac{R}{\omega \cdot L}\right)=\gamma \cdot i_{p} \cdot \sin (\omega \cdot t-\alpha)=\gamma \cdot i\left(\frac{t}{\gamma}\right) \\
& i^{\prime}\left(t^{\prime}\right)=i_{p}^{\prime} \cdot \sin \left(\omega^{\prime} \cdot t^{\prime}+\alpha^{\prime}\right)=\sqrt{\left(\frac{1}{R^{\prime}}\right)^{2}+\left(\omega^{\prime} \cdot C^{\prime}\right)^{2}} \cdot e_{p}^{\prime} \cdot \sin \left(\omega^{\prime} \cdot t^{\prime}+\operatorname{arctg} \omega^{\prime} \cdot R^{\prime} \cdot C^{\prime}\right)= \\
& =\gamma \cdot \sqrt{\left(\frac{1}{R}\right)^{2}+(\omega \cdot C)^{2}} \cdot e_{p} \cdot \sin (\omega \cdot t+\operatorname{arctg} \omega \cdot R \cdot C)==\gamma \cdot i_{p} \cdot \sin (\omega \cdot t+\alpha)=\gamma \cdot i\left(\frac{t}{\gamma}\right)
\end{aligned}
$$

The magnitudes of the admittances of the circuits presented in Fig. 11 and Fig. 12 in both coordinate systems $S$ ' and $S$ preserve their values:

$$
\begin{aligned}
& y^{\prime}=\sqrt{\left(\frac{1}{R^{\prime}}\right)^{2}+\left(\frac{1}{\omega^{\prime} \cdot L^{\prime}}\right)^{2}}=\sqrt{G^{\prime 2}+B_{L^{\prime}}^{\prime 2}}=\sqrt{\left(\frac{1}{R}\right)^{2}+\left(\frac{1}{\omega \cdot L}\right)^{2}}=\sqrt{G^{2}+B_{L}^{2}}=y \\
& y^{\prime}=\sqrt{\left(\frac{1}{R^{\prime}}\right)^{2}+\left(\omega^{\prime} \cdot C^{\prime}\right)^{2}}=\sqrt{G^{\prime 2}+B_{C^{\prime}}^{\prime 2}}=\sqrt{\left(\frac{1}{R}\right)^{2}+(\omega \cdot C)^{2}}=\sqrt{G^{2}+B_{C}^{2}}=y
\end{aligned}
$$

The triangles of the magnitudes of the admittances of both circuits (Fig. 13 and Fig. 14) preserve their forms and scales in the coordinate systems $S$ ' and $S$. 


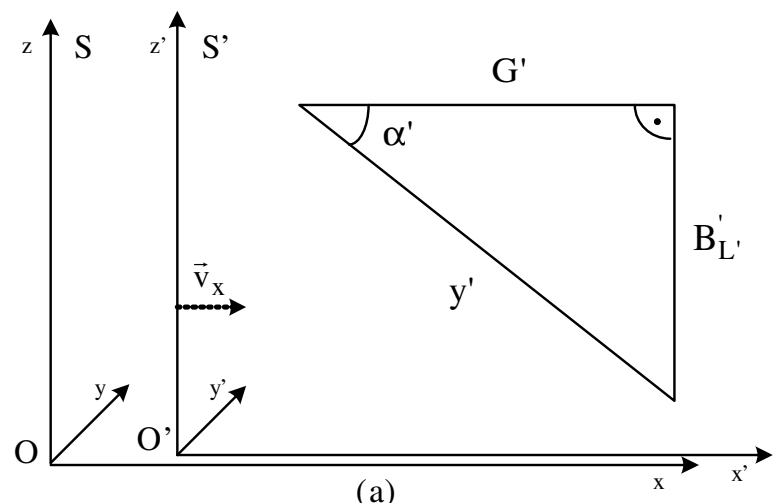

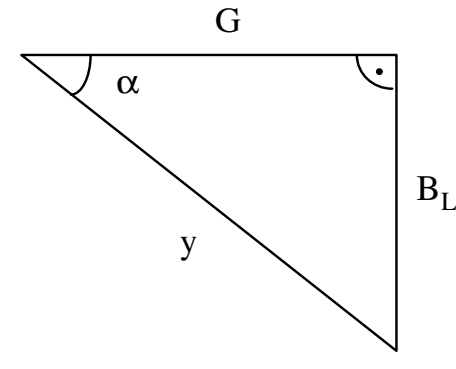

(b)

Fig. 13. Triangles of the admittances of a moving parallel RL electric circuit with a uniform speed $\vec{v}_{x}$ towards a static Cartesian coordinate system $S$.

The equivalent circuits of the networks, given in Fig. 11 and Fig. 12, in phasor form are presented in Fig. 15 and Fig. 16.

The vector diagrams of both circuits are presented in Fig. 17 and Fig. 18. These vector diagrams present Kirchhoff's current law in phasor form graphically:

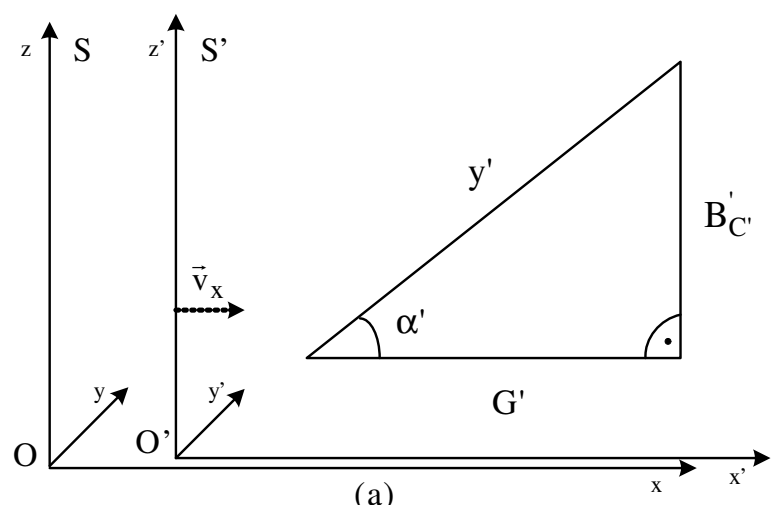

(a)

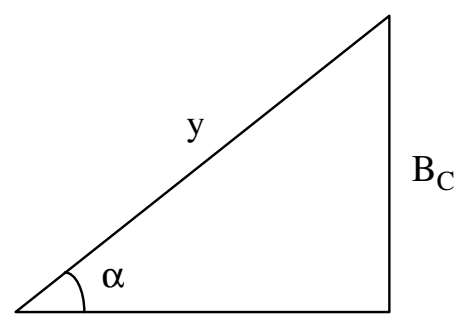

G

(b)

Fig. 14. Triangles of the admittances of a moving parallel RC electric circuit with a uniform speed $\vec{v}_{x}$ towards a static Cartesian coordinate system $S$.

$$
\begin{aligned}
& \dot{I^{\prime}}=\dot{I}_{R^{\prime}}+\dot{I}^{\prime} L^{\prime}=\gamma \cdot \dot{I}_{R}+\gamma \cdot \dot{I}_{L}=\gamma \cdot \dot{I} \\
& \dot{I^{\prime}}=\dot{I}_{R^{\prime}}+\dot{I}_{I^{\prime}}=\gamma \cdot \dot{I}_{R}+\gamma \cdot \dot{I}_{C}=\gamma \cdot \dot{I}
\end{aligned}
$$



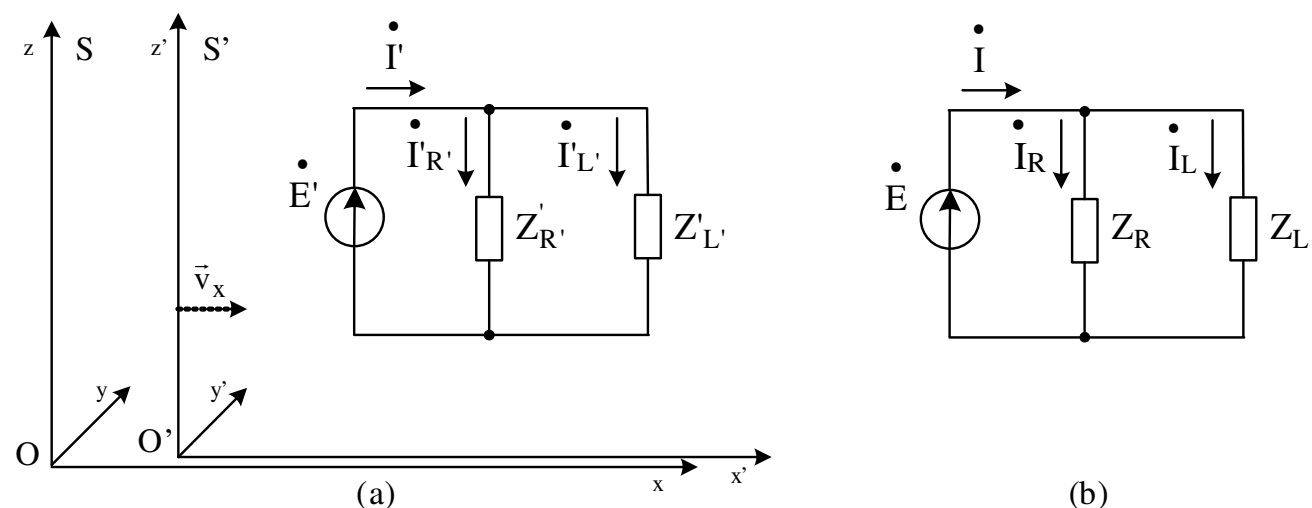

(b)

Fig. 15. Equivalent circuit of a moving parallel RL electric circuit with a uniform speed $\vec{v}_{x}$ towards a static Cartesian coordinate system $S$ according to the phasor approach.
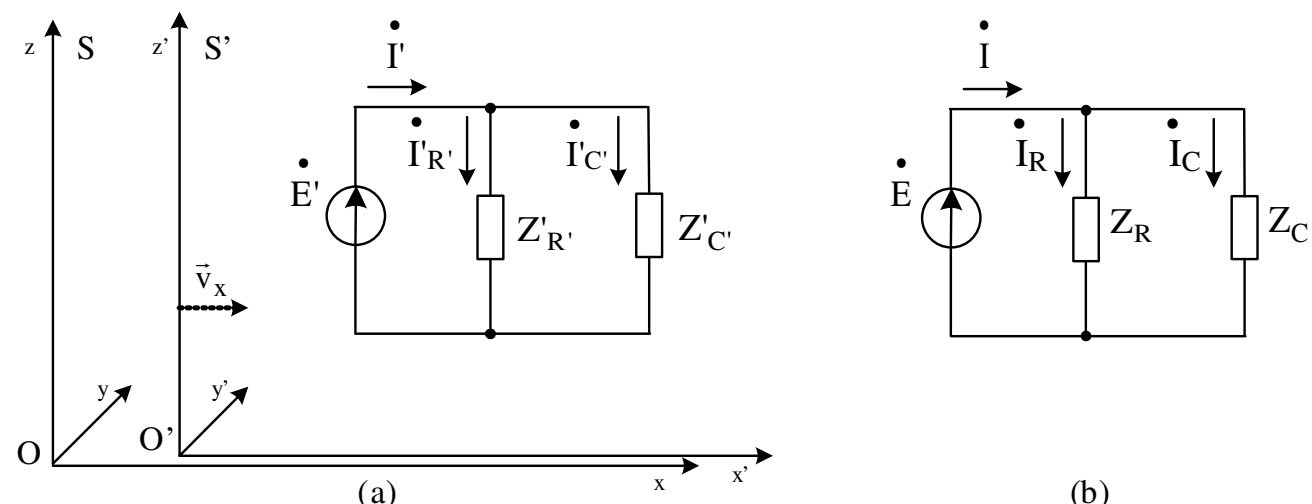

(b)

Fig. 16. Equivalent circuit of a moving parallel RC electric circuit with a uniform speed $\vec{v}_{x}$ towards a static Cartesian coordinate system $S$ according to the phasor approach.
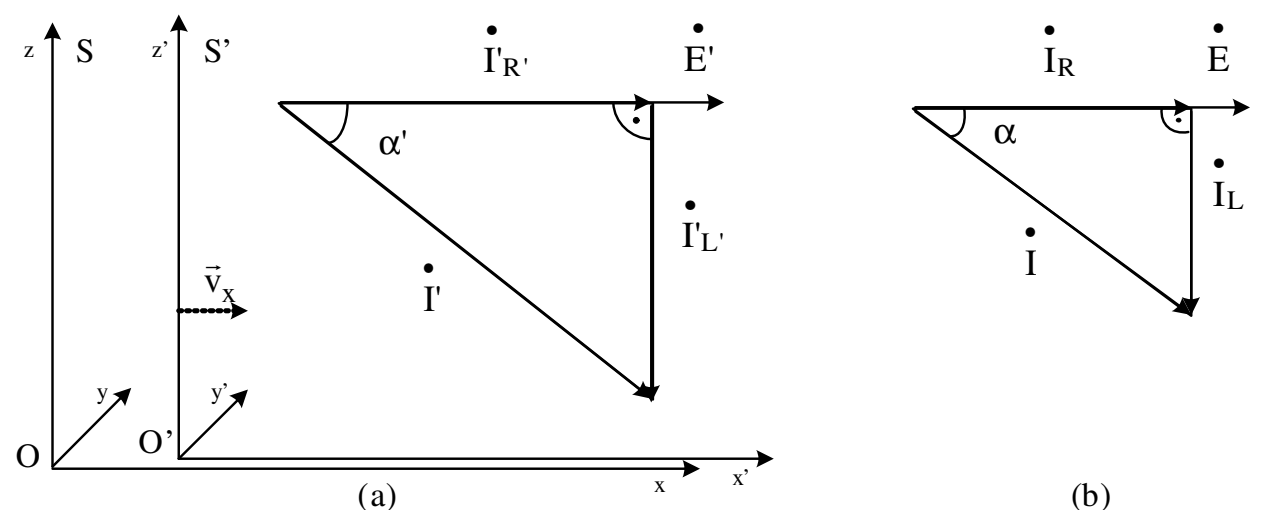

(b)

Fig. 17. Vector diagram of a moving parallel RL electric circuit with a uniform speed $\vec{v}_{x}$ towards a static Cartesian coordinate system $S$ according to the phasor approach. 


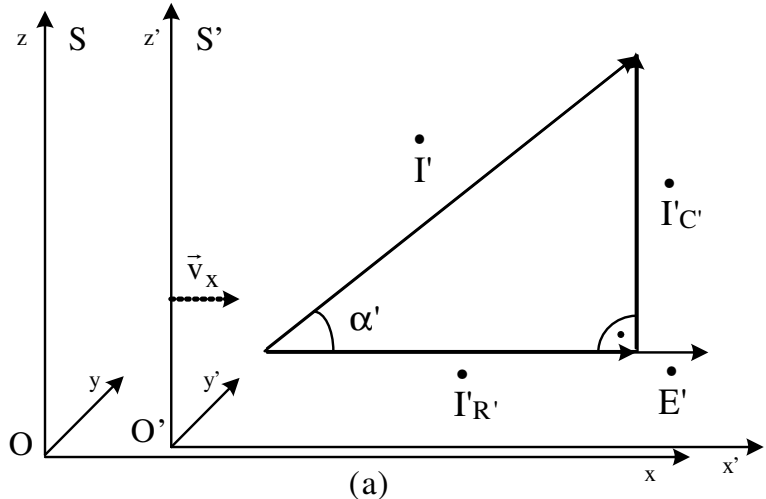

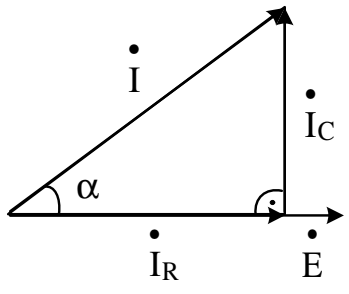

(b)

Fig. 18. Vector diagram of a moving parallel RC electric circuit with a uniform speed $\vec{v}_{x}$ towards a static Cartesian coordinate system $S$ according to the phasor approach.

\subsection{Resonance in fast moving $\mathrm{AC}$ linear electric circuits}

In Fig. 19 a serial RLC electric circuit operating in harmonic regime is presented, and in Fig. 20 a parallel RLC circuit is given, too.

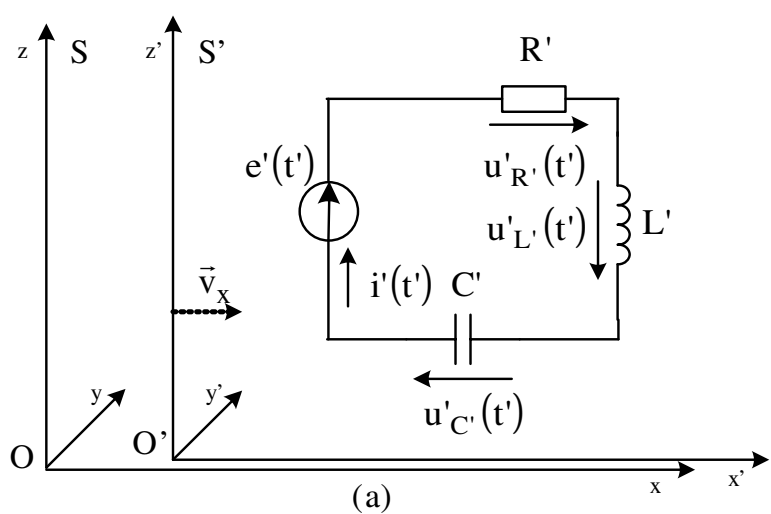

(a)

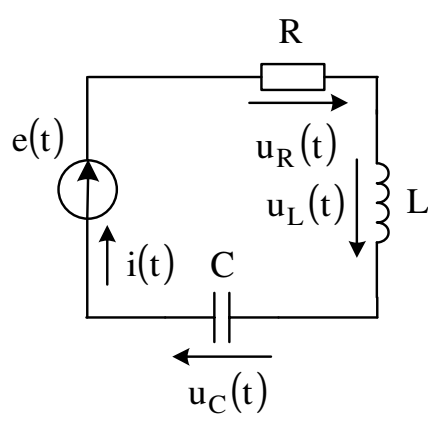

(b)

Fig. 19. A moving serial RLC electric circuit with a uniform speed $\vec{v}_{x}$ towards a static Cartesian coordinate system $S$.

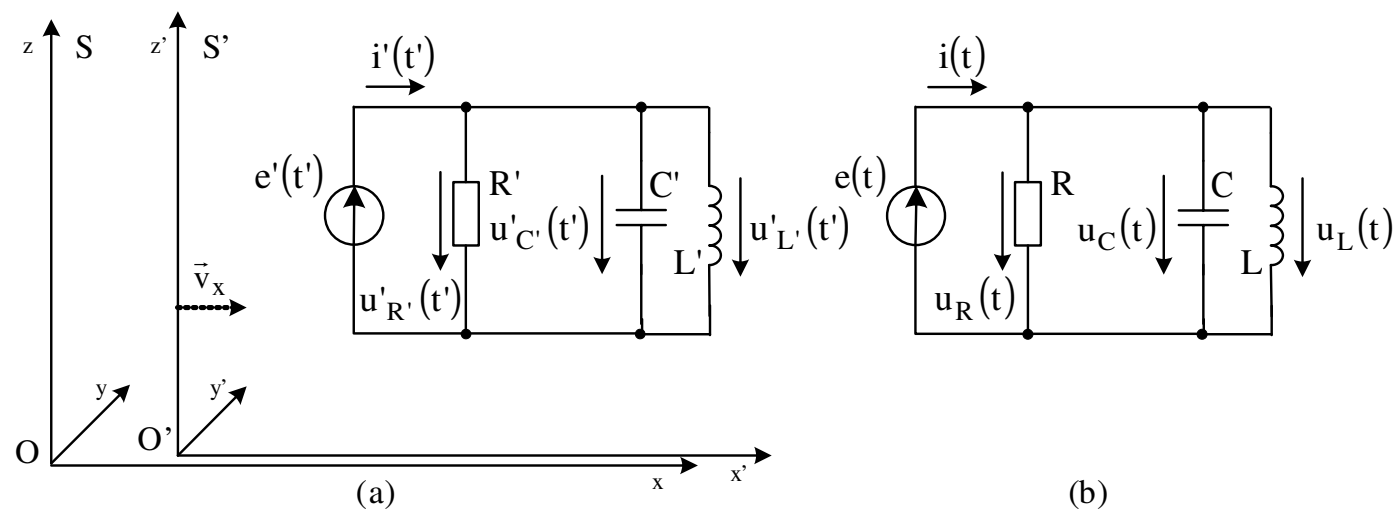

Fig. 20. A moving parallel RLC electric circuit with a uniform speed $\vec{v}_{x}$ towards a static Cartesian coordinate system $S$. 
The angular frequency was already presented by equation (39) and it was shown that the result corresponds to that from the transverse Doppler effect (Kittel, 1963, p. 344). The relation among the resonant frequencies $\omega_{r}^{\prime}$ and $\omega_{r}$ in both coordinate systems $S$ ' and $S$ in case of these two electric circuits can be extracted by the Thompson's formula:

$$
\omega_{r}{ }^{\prime}=\frac{1}{\sqrt{L^{\prime} \cdot C^{\prime}}}=\frac{1}{\sqrt{\frac{L}{\gamma} \cdot \frac{C}{\gamma}}}=\gamma \cdot \frac{1}{\sqrt{L \cdot C}}=\gamma \cdot \omega_{r}
$$

It confirms the result received by equation (39).

The relations among the wave resistances $\rho$ ' and $\rho$ and the quality factors $Q$ ' and $Q$ in both coordinate systems $S^{\prime}$ and $S$ of the serial RLC circuit are as follows:

$$
\begin{gathered}
\rho^{\prime}=X_{L_{r}^{\prime}}^{\prime}=\omega_{r}^{\prime} \cdot L^{\prime}=\gamma \cdot \omega_{r} \cdot \frac{L}{\gamma}=\omega_{r} \cdot L=X_{L_{r}}=X_{C_{r}^{\prime}}^{\prime}=\frac{1}{\omega_{r}^{\prime} \cdot C^{\prime}}=\frac{1}{\gamma \cdot \omega_{r} \cdot \frac{C}{\gamma}}=\frac{1}{\omega_{r} \cdot C}=X_{C_{r}}=\rho \\
Q^{\prime}=\frac{\rho^{\prime}}{R^{\prime}}=\frac{\omega_{r}^{\prime} \cdot L^{\prime}}{R^{\prime}}=\frac{1}{\omega_{r}^{\prime} \cdot R^{\prime} \cdot C^{\prime}}=\frac{\omega_{r} \cdot L}{R}=\frac{1}{\omega_{r} \cdot R \cdot C}=\frac{\rho}{R}=Q
\end{gathered}
$$

The relations among the wave conductances $\delta$ ' and $\delta$ and the quality factors $Q$ ' and $Q$ in both coordinate systems $S$ ' and $S$ of the parallel RLC circuit are as follows:

$$
\begin{gathered}
\delta^{\prime}=B_{L_{r}^{\prime}}^{\prime}=\frac{1}{\omega_{r}^{\prime} \cdot L^{\prime}}=\frac{1}{\gamma \cdot \omega_{r} \cdot \frac{L}{\gamma}}=\frac{1}{\omega_{r} \cdot L}=B_{L_{r}}=B_{C^{\prime} r}^{\prime}=\omega_{r}^{\prime} . C^{\prime}=\gamma \cdot \omega_{r} \cdot \frac{C}{\gamma}=\omega_{r} \cdot C=B_{C_{r}}=\delta \\
Q^{\prime}=\frac{\delta^{\prime}}{G^{\prime}}=\frac{\delta^{\prime}}{\frac{1}{R^{\prime}}}=\frac{R^{\prime}}{\omega_{r}^{\prime} \cdot L^{\prime}}=\omega_{r}^{\prime} \cdot R^{\prime} \cdot C^{\prime}=\frac{R}{\omega_{r} \cdot L}=\omega_{r} \cdot R \cdot C=\frac{\delta}{\frac{1}{R}}=\frac{\delta}{G}=Q
\end{gathered}
$$

So, the wave resistances, the wave conductances and the quality factors preserve their values in both coordinate systems.

\subsection{Transient processes in fast moving linear electric circuits}

Some of the interesting regimes in the fast moving linear electric circuits are connected with the transient processes. In Fig. 21 a first order RL linear electric circuits is presented, and in Fig. 22 a first order RC linear electric circuit is given, too. If the commutations occur at the moment $t^{\prime}=0$ in the coordinate system $S^{\prime}$, then the currents in the coordinate systems $S$ and $S^{\prime}$ will be aperiodic and they will have the following forms: 
$i^{\prime}\left(t^{\prime}\right)=i_{\text {steady } y \text { state }}^{\prime}+\left[i^{\prime}(0+)-i_{\text {stead } y-\text { state }}^{\prime}(0+)\right] \cdot e^{-\frac{t^{\prime}}{\tau^{\prime}}}=i_{\text {steady } y \text { state }}^{\prime}+A^{\prime} \cdot e^{-k^{\prime} t^{\prime}}=\frac{e_{1}^{\prime}}{R^{\prime}}+\left(0-\frac{e_{1}^{\prime}}{R^{\prime}}\right) \cdot e^{-\frac{R^{\prime} \cdot t^{\prime}}{L^{\prime}}}=$

$=\frac{\gamma \cdot e_{1}}{R}+\left(0-\frac{\gamma \cdot e_{1}}{R}\right) \cdot e^{-\frac{{ }^{R} \cdot \frac{t}{\gamma}}{\gamma}}=\frac{\gamma \cdot e_{1}}{R}+\left(0-\frac{\gamma \cdot e_{1}}{R}\right) \cdot e^{-\frac{R \cdot t}{L}}=\gamma \cdot i_{\text {stead } y-\text { state }}+\left[\gamma \cdot i(0+)-\gamma \cdot i_{\text {steady } y \text { state }}(0+)\right] \cdot e^{-\frac{t}{\tau}}=(67)$

$=\gamma \cdot i_{\text {steady-state }}+\gamma \cdot A \cdot e^{-k . t}=\gamma \cdot i\left(\frac{t}{\gamma}\right)$

$i^{\prime}\left(t^{\prime}\right)=i_{\text {steady-state }}^{\prime}+\left[i^{\prime}(0+)-i_{\text {steady }- \text { state }}^{\prime}(0+)\right] e^{-\frac{t^{\prime}}{\tau^{\prime}}}=i_{\text {steady }- \text { state }}^{\prime}+A^{\prime} \cdot e^{-k^{\prime} \cdot t^{\prime}}=0+\left(\frac{e_{1}^{\prime}}{R^{\prime}}-0\right) \cdot e^{-\frac{t^{\prime}}{R^{\prime} \cdot C^{\prime}}}=$

$=0+\left(\frac{\gamma \cdot e_{1}}{R}-0\right) \cdot e^{-\frac{\frac{t}{\gamma}}{R \cdot \frac{C}{\gamma}}}=0+\left(\frac{\gamma \cdot e_{1}}{R}-0\right) \cdot e^{-\frac{t}{R \cdot C}}=\gamma \cdot i_{\text {steady }- \text { state }}+\left[\gamma \cdot i(0+)-\gamma \cdot i_{\text {steady }- \text { state }}(0+)\right] \cdot e^{-\frac{t}{\tau}}=(68)$

$=\gamma \cdot i_{\text {steady }- \text { state }}+\gamma \cdot A \cdot e^{-k . t}=\gamma \cdot i\left(\frac{t}{\gamma}\right)$

Here, $i_{\text {steady-state }}^{\prime}$ and $i_{\text {steady-state }}$ are the steady state values of the flowing currents; $i^{\prime}(0+), i(0+)$, $i_{\text {steady-state }}^{\prime}(O+)$ and $i_{\text {steady-state }}(0+)$ are the initial conditions (the initial values of the currents) after the commutation; $k^{\prime}=-\frac{1}{\tau^{\prime}}$ and $k=-\frac{1}{\tau}$ are the roots of the characteristic equations of the explored circuits. It is clear that the time constants $\tau^{\prime}$ and $\tau$ in both coordinate systems $S^{\prime}$ and $S$ are connected with the following relations:

$$
\begin{gathered}
\tau^{\prime}=\frac{L^{\prime}}{R^{\prime}}=\frac{\frac{L}{\gamma}}{R}=\frac{L}{\gamma \cdot R}=\frac{\tau}{\gamma} \\
\tau^{\prime}=R^{\prime} \cdot C^{\prime}=R \cdot \frac{C}{\gamma}=\frac{R \cdot C}{\gamma}=\frac{\tau}{\gamma} \\
k^{\prime}=-\frac{1}{\tau^{\prime}}=-\frac{1}{\frac{\tau}{\gamma}}=-\frac{\gamma}{\tau}=\gamma \cdot k
\end{gathered}
$$

In Fig. 23 a second order serial RLC linear electric circuit is presented. In such a circuit three different transient processes are possible depending on the values of the roots of the characteristic equation of the circuit $k_{1}^{\prime}$ and $k_{2}^{\prime}$.

If the roots are: $k_{1}^{\prime} \neq k_{2}^{\prime} ; k_{1}^{\prime}, k_{2}^{\prime} \in R e ; k_{1}^{\prime}<0, k_{2}^{\prime}<0$, then the transient process is overdamped:

$$
\begin{aligned}
& i^{\prime}\left(t^{\prime}\right)=i_{\text {steady-state }}^{\prime}+A_{1}^{\prime} \cdot e^{k_{1}^{\prime} \cdot t^{\prime}}+A_{2}^{\prime} \cdot e^{k_{2}^{\prime} \cdot t^{\prime}}=\gamma \cdot i_{\text {steady-state }}+\gamma \cdot A_{1} \cdot e^{\gamma \cdot k_{1} \cdot \frac{t}{\gamma}}+\gamma \cdot A_{2} \cdot e^{\gamma \cdot k_{2} \cdot \frac{t}{\gamma}}= \\
& =-\frac{C^{\prime} \cdot e_{1}^{\prime} \cdot k_{1}^{\prime} \cdot k_{2}^{\prime}}{k_{2}^{\prime}-k_{1}^{\prime}} \cdot e^{k_{1}^{\prime} \cdot t^{\prime}}+\frac{C^{\prime} \cdot e_{1}^{\prime}{ }_{1} k_{1}^{\prime} \cdot k_{2}^{\prime}}{k_{2}^{\prime}-k_{1}^{\prime}} \cdot e^{k_{2}^{\prime} \cdot t^{\prime}}=-\frac{\gamma \cdot C \cdot e_{1} \cdot k_{1} \cdot k_{2}}{k_{2}-k_{1}} \cdot e^{k_{1} \cdot t}+\frac{\gamma \cdot C \cdot e_{1} \cdot k_{1} \cdot k_{2}}{k_{2}-k_{1}} \cdot e^{k_{2} \cdot t}= \\
& =\gamma \cdot i_{\text {steady-state }}+\gamma \cdot A_{1} \cdot e^{k_{1} \cdot t}+\gamma \cdot A_{2} \cdot e^{k_{2} \cdot t}=\gamma \cdot i\left(\frac{t}{\gamma}\right)
\end{aligned}
$$



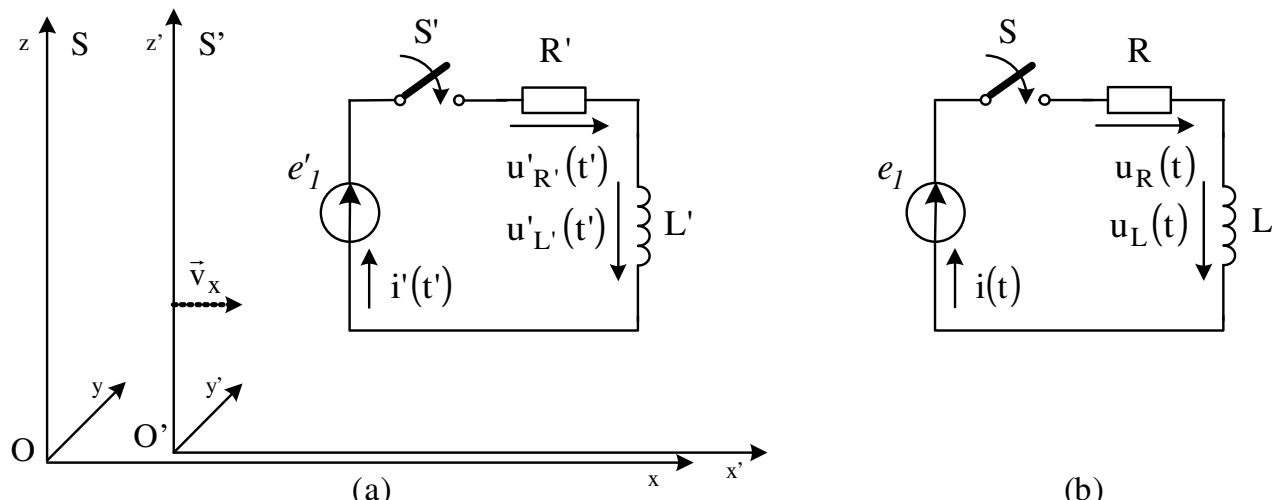

(b)

Fig. 21. A moving first order RL electric circuit with a uniform speed $\vec{v}_{x}$ towards a static Cartesian coordinate system $S$.

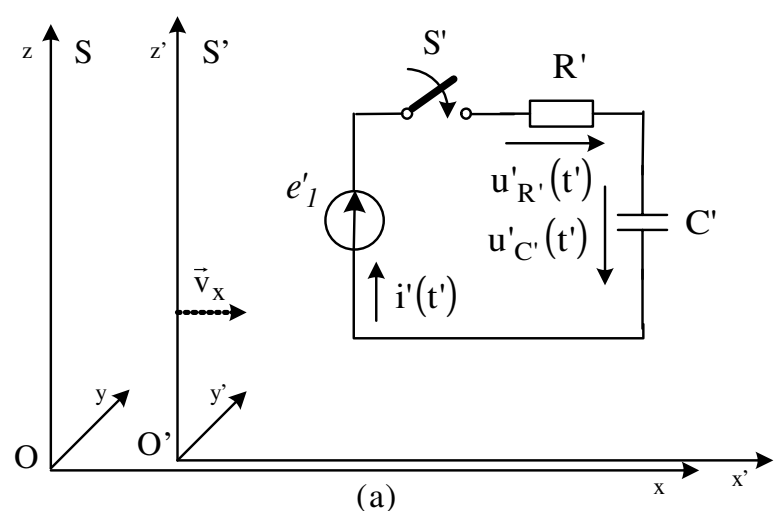

(a)

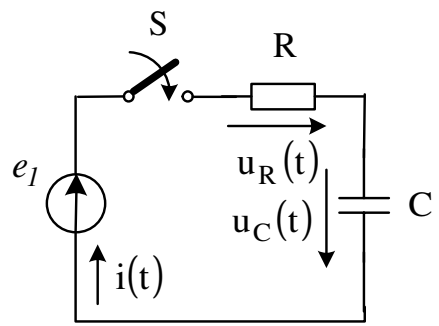

(b)

Fig. 22. A moving first order RC electric circuit with a uniform speed $\vec{v}_{x}$ towards a static Cartesian coordinate system $S$.

If the roots are: $k_{1}^{\prime}=k_{2}^{\prime} ; k_{1}^{\prime}, k_{2}^{\prime} \in R e ; k_{1}^{\prime}<0, k_{2}^{\prime}<0$, then the transient process is critically damped:

$$
\begin{aligned}
& i^{\prime}\left(t^{\prime}\right)=i_{\text {steady-state }}^{\prime}+A_{1}^{\prime} \cdot e^{k_{1}^{\prime} \cdot t^{\prime}}+A_{2}^{\prime} \cdot t^{\prime} \cdot e^{k_{1}^{\prime} \cdot t^{\prime}}=\gamma \cdot i_{\text {steady-state }}+\gamma \cdot A_{1} \cdot e^{\gamma \cdot k_{1} \cdot \frac{t}{\gamma}}+\gamma^{2} \cdot A_{2} \cdot \frac{t}{\gamma} \cdot e^{\gamma \cdot k_{1} \cdot \frac{t}{\gamma}}= \\
& =C^{\prime} \cdot e_{1}^{\prime} \cdot k_{1}^{\prime 2} \cdot t^{\prime} \cdot e^{k_{1} \cdot t^{\prime}}=\gamma \cdot C \cdot e_{1} \cdot k_{1}^{2} \cdot t \cdot e^{k_{1} \cdot t}=\gamma \cdot i_{\text {steady-state }}+\gamma \cdot A_{1} \cdot e^{k_{1} \cdot t}+\gamma \cdot A_{2} \cdot t \cdot e^{k_{1} \cdot t}=\gamma \cdot \cdot\left(\frac{t}{\gamma}\right)
\end{aligned}
$$

If the roots are: $k_{1,2}^{\prime}=-\beta^{\prime} \pm j \Omega^{\prime} ; k_{1}^{\prime}, k_{2}^{\prime} \in \mathrm{Compl}$, then the transient process is underdamped:

$$
\begin{aligned}
& i^{\prime}\left(t^{\prime}\right)=i_{\text {steady-state }}^{\prime}+A^{\prime} \cdot e^{-\beta^{\prime} \cdot t^{\prime}} \cdot \sin \left(\Omega^{\prime} \cdot t^{\prime}+\mu^{\prime}\right)=\gamma \cdot i_{\text {steady-state }}+\gamma \cdot A \cdot e^{-\gamma \cdot \beta \cdot \frac{t}{\gamma}} \cdot \sin \left(\gamma \cdot \Omega \cdot \frac{t}{\gamma}+\mu\right)= \\
& =\frac{e_{1}^{\prime}}{\Omega^{\prime} \cdot L^{\prime}} \cdot e^{-\beta^{\prime} \cdot t^{\prime}} \cdot \sin \left(\Omega \cdot{ }^{\prime} \cdot t^{\prime}\right)=\frac{\gamma \cdot e_{1}}{\Omega \cdot L} \cdot e^{-\beta \cdot t} \cdot \sin (\Omega \cdot t)=\gamma \cdot i_{\text {steady-state }}+\gamma \cdot A \cdot e^{-\beta \cdot t} \cdot \sin (\Omega \cdot t+\mu)=\gamma \cdot i\left(\frac{t}{\gamma}\right)
\end{aligned}
$$

Here, $\beta^{\prime}$ and $\beta$ are damping coefficients; $\Omega^{\prime}$ and $\Omega$ - natural angular frequencies; $\mu$ ' and $\mu$ - phase shifts. Here, it is obvious, that:

$$
k_{1,2}^{\prime}=\gamma \cdot k_{1,2}
$$




$$
\begin{aligned}
& \beta^{\prime}=\gamma \cdot \beta \\
& \Omega^{\prime}=\gamma \cdot \Omega \\
& \mu^{\prime}=\mu
\end{aligned}
$$

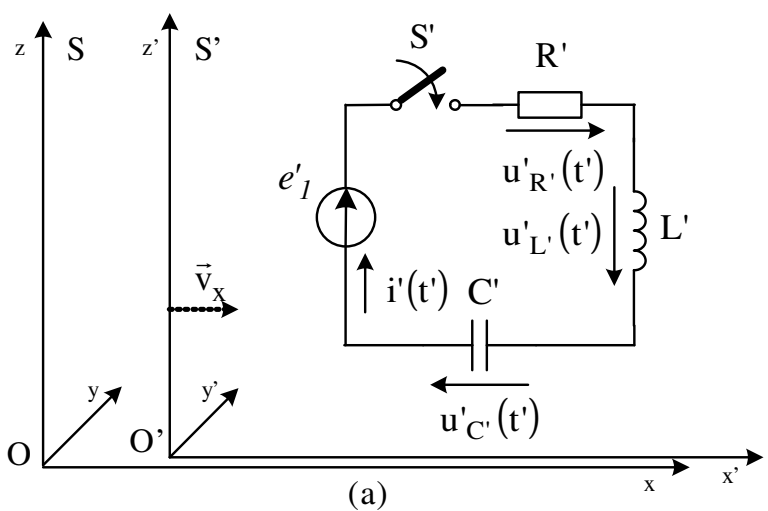

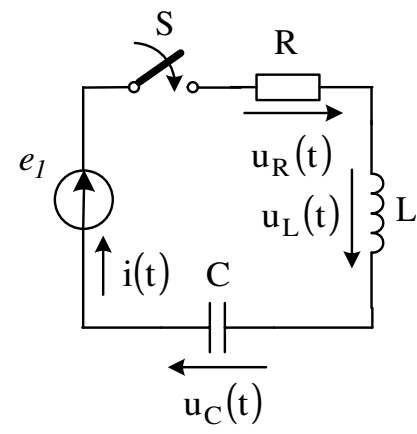

(b)

Fig. 23. A moving second order RLC electric circuit with a uniform speed $\vec{v}_{x}$ towards a static Cartesian coordinate system $S$.

\section{Conclusions}

As a result of the research, the basic laws (Kirchhoff's current law, Kirchhoff's voltage law, Ohm's law, Joule's law and the energy conservation law) in relativistic form for fast moving electric circuits, operating in AC regimes, were extracted. The relativistic forms of the phasors of the basic quantities of the electric circuits (currents, voltages), the complex powers and the relativistic relations of the basic parameters of the circuits (angular frequencies, phases, phase shifts, reactances, susceptances, impedances, admittances) were presented, too. Additionally, resonances and transient processes in fast moving linear electric circuits were observed, as well. The corresponding formulas were extracted step by step in order to show the way of reasoning. All that is an essential part of the RCT, which additional research can be very useful for exploration of the EM processes in fast moving electric circuits...

\section{References}

Einstein, A. (1905). Zur elektrodynamik bewegter körper. Annalen der Physik, 17, 891-921. (in German). https://doi.org/10.1002/andp.19053221004

Einstein, A. Laub, J. (1908). Über die elektromagnetischen grundgleihungen für bewegte körper. Annalen der Physik, 331(8), 532-540. (in German). https://doi.org/10.1002/andp.19083310806

Feynmann, R., Leighton, R., Sands, M. (1964a). The Feynmann lectures on physics. vol. 1, Ad-dison Wesley Publishing House, Inc.

Feynmann, R., Leighton, R., Sands, M. (1964b). The Feynmann llectures on physics. vol. 2, Ad-dison Wesley Publishing House, Inc.

Hertz, H. (1890). Ueber die grundgleichungen der electrodynamik füruhende körper. Wied. Ann., 41, 106-149. (in German)

Kittel, C., Knight, W. D., Ruderman, M. A. (1963). Mechanics, Berkeley physics course, vol. I, N. Y., McGraw-Hill Book Co. 
Maxwell, J. C. (1861). On physical lines of force. Philosophical Magazine and Journal of Sci-ence, Part I, 161-175. Part II, 281-291, 338-347 (March). Part III, 12-24, (April, May). Part IV, 8595. https://doi.org/10.1080/14786446108643067

Maxwell, J. C. (1865). A Dynamical theory of the electromagnetic field. Philosophical Transac-tions of the Royal Society of London, 155, 459-512. https://doi.org/10.1098/rstl.1865.0008

Maxwell, J. C. (1873). A Treatise on electricity and magnetizm. vol. I, vol. II, London, Macmil-lan and co.

Meerovich, E. A. (1966). Metody reliativistkoy elektrodinamiky v elektrotehnike. Moskva, Ener-gia. (in Russian)

Meerovich, E. A., Meierovich, B. E. (1987). Metody reliativistkoy elektrodinamiky v el-ektrotehnike i elektrofizike. Moskva, Energoatomizdat. (in Russian)

Panov, E. I. (2015). Rotary theory of the magnetic field, basics, concepts, methods and models. (monograph), Germany, LAP Lambert Academic Publishing, 148 pages. ISBN 978-3-65978098-1

Panov, E. I. (2017a). On the electrodynamics of moving bodies according to the rotary theory. Proceedings of the Second International Scientific Conference "Intelligent Information Technologies for Industry" (IITI'17), Advances in Intelligent Systems and Computing 680, 2, Varna, Bulgaria, 280-289. Springer, (SCOPUS), ISSN 2194-5357, ISSN 2194-5365 (electronic), ISBN 978-3-319-68323-2, ISBN 978-3-319-68324-9 (eBook), https://doi.org/10.1007/978-3319-68324-9_31

Panov, E. I. (2017b). On another proof of the formula $\mathrm{E}=\mathrm{m} . \mathrm{c} 2$ according to the rotary theory, Annual Journal of the Technical University of Varna, 1(1), 13-20. ISSN 2603-316 (Online), https://doi.org/10.29114/ajtuv.vol1.iss1.22

Panov, E. I. (2018). Direct current regimes in the linear electric circuits according to the relativ-istic circuit theory, Annual Journal of the Technical University of Varna, 2(1), 17-29. ISSN 2603316X (Online), https://doi.org/10.29114/ajtuv.vol2.iss1.66

Pauli, W. Jr. (1958). Theory of relativity. London, Pergamon Press.

Polivanov, K. M. (1982). Elektrodinamika dvijushtihsia tel. Moskva, Energoizdat. (in Russian)

Purcell, E. M. (1965). Electricity and magnetism, Berkeley physics course. vol. II, N. Y., McGrawHill Book Co.

Simonyi, K. (1964). Teoreticheskaia elektrotehnika. Moskva, Mir. (in Russian)

Smythe, W. R. (1989). Static and dynamic electricity. Third Edition, Hemisphere Publishing Corporation. 